HIFAN 1772

An estimate of collisional beam scattering during final focus in NDCX-II

\author{
by \\ R.H. Cohen \\ Lawrence Livermore National Laboratory
}

\begin{abstract}
HIFS-VNL
Lawrence Berkeley National Laboratory (on behalf of U.S. HIFS-VNL)

1 Cyclotron Road, Berkeley, CA 94720
\end{abstract}

\author{
Accelerator Fusion Research Division \\ Ernest Orlando Lawrence Berkeley National Laboratory \\ University of California \\ Berkeley, California 94720
}

March 2010

This document was prepared as an account of work sponsored by the United States Government. While this document is believed to contain correct information, neither the United States Government nor any agency thereof, nor The Regents of the University of California, nor any of their employees, makes any warranty, express or implied, or assumes any legal responsibility for the accuracy, completeness, or usefulness of any information, apparatus, product, or process disclosed, or represents that its use would not infringe privately owned rights. Reference herein to any specific commercial product, process, or service by its trade name, trademark, manufacturer, or otherwise, does not necessarily constitute or imply its endorsement, recommendation, or favoring by the United States Government or any agency thereof, or The Regents of the University of California. The views and opinions of authors expressed herein do not necessarily state or reflect those of the United States Government or any agency thereof or The Regents of the University of California.

This work was supported by the Director, Office of Science, Office of Fusion Energy Sciences, of the U.S. Department of Energy under Contract No. DE-AC02-05CH11231. 


\section{An estimate of collisional beam scattering during final focus in NDCX-II*}

R.H. Cohen, LLNL and HIFS-VNL, 3/23/10

The final focus of NDCX-II contains a region with quite high plasma density. We estimate here how much collisional scatter we expect from transit through this plasma. A separate question, not explored here, is how much scatter there might be off of collective fluctuations in the neutralizing plasma, including those driven by the passage of the beam.

From the NRL tables the rate of perpendicular scatter is given by:

$$
\frac{d}{d t}\left(v_{\perp}-\bar{v}_{\perp}\right)^{2}=\nu_{\perp} v^{2}
$$

where the relevant scattering process is fast beam ions scattering off of the ions and electrons in the background plasma. For MeV-plus-energy beam ions and a few eV plasma electrons (and plasma ions), we are in the limit of fast test particles scattering on a slow background, so that

$$
\nu_{\perp}=1.8 \times 10^{-7}\left(n_{e} \lambda_{i e}+n_{i} \lambda_{i i} Z_{i}^{2}\right) Z_{b}^{2} \mu_{b}^{-1 / 2} \epsilon_{b}^{-3 / 2}
$$

Taking $v^{2}=$ constant $=4 v_{b}^{2}$ in the final focus region, the net integrated r.m.s. scattering angle $\theta_{s}$ is then given by

$$
\theta_{s}=\left(v_{b}^{-1} \int \nu_{\perp} d z\right)^{1 / 2}
$$

and we would like the integrated spread of spot size resulting from this scattering to be small compared to the beam size; hence we compare

$$
\Delta r_{s}=\int d z v_{r} / v_{z} \approx \int d z \theta_{s} \approx v_{b}^{-1 / 2} \int d z\left(\int^{z} \nu_{\perp} d z^{\prime}\right)^{1 / 2}
$$

to the spot size neglecting scattering effects. The details, as expected, depend on the distribution of plasma density, which, because of the planned injection location and magneticfield design, will be high near the target and fall off upstream.

To obtain an estimate, we approximate the plasma density as uniform, with value $n_{e p}$ over a length $L_{s}$, and present expressions relative to reference values of $n_{e p}=10^{15}$ $\mathrm{cm}^{-3}$ and $L_{s}=50 \mathrm{~cm}$. For fast beam ions, the electron and ion plasma temperatures and the plasma ion atomic mass enter only very weakly via the Coulomb logarithm, which we evaluate here for plasma temperatures $T_{e}=T_{i}=3 \mathrm{eV}$ and ion mass $A_{i}=27$ (Aluminum), and beam energy $2 \mathrm{MeV}$, beam ion mass 7 (Lithium), and charge 1. For these parameters we obtain $\log \lambda_{e i}=23$ and $\log \lambda_{i i}=22$, and hence take simply 23 for the coulomb log. Then we obtain

$$
\begin{aligned}
\Delta r_{s} \approx & \frac{2}{3}\left(\nu_{\perp} / v_{b}\right)^{1 / 2} L_{s}^{3 / 2}= \\
& 3 \times 10^{-3} \mathrm{~cm} \times\left(\frac{n_{e} \lambda_{i e}+n_{i} \lambda_{i i} Z_{i}^{2}}{2 \times 23 \times 10^{14} \mathrm{~cm}^{-3}}\right)^{1 / 2} \times \frac{2 \mathrm{MeV}}{\epsilon_{b}} \times\left(\frac{L_{s}}{50 \mathrm{~cm}}\right)^{3 / 2}
\end{aligned}
$$

Since the desired focussed spot size is around $0.1 \mathrm{~cm}$, we conclude that collisional scattering is a negligible concern for the likely range of plasma densities and scale lengths.

* Work performed by LLNL for U.S.D.O.E. under contract DE-AC52-07NA27344. 